\title{
A New Image Quality Index and it's Application on MRI Image
}

\author{
Md. Tariqul Islam \\ Dept. of Electronics and Communication Engineering, Khulna University of Engineering \& Technology, Khulna, 9203, \\ Bangladesh \\ Email: mti.tariqul12@gmail.com
}

\author{
Sheikh Md. Rabiul Islam \\ Dept. of Electronics and Communication Engineering, Khulna University of Engineering \& Technology, Khulna, 9203, \\ Bangladesh \\ Email: robi@ece.kuet.ac.bd
}

Received: 29 November 2020; Accepted: 26 February 2021; Published: 08 August 2021

\begin{abstract}
Image quality assessment (IQA) is a process of measurement of the image quality using the evaluations of subjective value with the model of computation. The quality of the image can be calculated by using different types of method where each method works with using isolated features of image. One very renowned method is structural similarity index (SSIM) which measured the quality of image comparing structure of image and the structure stage is obtained from pixel-based stage. FSIM (Feature Similarity Index) measured image quality using low level feature and Gradient magnitude (GM) act as primary feature of image. In this work, a novel MFSIM (Moderate Feature Similarity Index) is introduced which work with full reference IQA, HVS (Human Visual System) and low-level feature of images. In MFSIM the Phase Congruency (PC) is used as primary feature where the PC is dimensionless contrast invariant. In the moderated FSIM the Gradient Magnitude (GM) of the image is considered as the feature of secondary. For application IQA, we applied into segmented image with original image using MRI images. The distortion level of the segmented image is calculated using different image quality index measurement techniques. The image can be used in numerous purposes and the quality of image is distorted for different reason. There are lots of applications where noise less of perfect image is used for getting exact result. So it is very important to find out the distortion level of image. For instance during the segmentation of MRI image for brain tumor detection, the exactness of image need to calculate so that the brain tumor can be find out accurately. So the main purpose of this research work is to introduce a new image quality index and find out the brain tumor and the segmented image quality.
\end{abstract}

Index Terms: IQA, MFSIM, SSIM, PC, GM, MRI.

\section{Introduction}

The quick increment of various technology and modern imaging system for communication, IQA has been getting an influential argument in a lot of infliction. The development of objective IQA metrics is essential for robustly and automatically measurement of image quality because the IQA subjective methods are unable to use in different aspect such as real time and automated systems. At the same time, it is predicted that the appraisal outcomes should be statistically compatible with the people observation. The main objective of this research work is to find out an authentic image quality index which provides more efficient result. Another objective is to find out brain tumor using segmentation and determine the segmented image quality. In the past decades, the research-based organization has developed numerous methods of IQA. In proportion with the presence of reference images FR (full reference), NR (no-reference), RR (reduced reference) are the classification of IQA metrics [1]. The traditional metrics for instance the PSNR and MSE perform straight on the image intensity and the performance of correlation between subjective fidelity ratings and them is not satisfactory. Consequently, various attempts have been applied on the manipulation of HVS with respect to IQA metrics. These types of models highlight the significance of HVS' reactivity to various [2] visual signals, for example Frequency Content, Contrast, Luminance as well as the interaction among signaling elements [3].

The procedure of structural similarity (SSIM) index is promoted by the requirement of capturing the disappearance of the image structure [4]. The structural information of the image visual scene is extracted by the highly adaption of HVS which is one of the speculations of SSIM. Consequently, a good estimation of recognized image quality should be provided by the measurement of structure similarity. MS-SSIM is obtained by the multi-scale extension of SSIM [5], 
bring out the result which is better than single-scale SSIM. However, the image visual information is showed redundant manner whereas an image is recognized by HVS using its low-level [6] features, for example edges and zero-crossings. On the other hand, the low-level [4] salient features of the image transport the significant information for the HVS to the scene interpretation. Correspondingly, the detectable change of the low-level features of image based on the observable deterioration of image, and for this reason a high standard IQA metric would be able to devised by the low-level feature comparison among distorted and reference images. After completing resolution, this work introduced Moderated Feature Similarity Index (MFSIM). In moderate feature similarity index the third component of image 'phase' is calculated and MFSIM is calculated by mixing magnitude, phase and frequency of the image. This index provides us better performance than the existing method.

With the modern technologies, different types of medical imaging techniques such as MRI, Ultrasound imaging, CT PET, X-ray Radiography are invented which are the visual presentation of the body internal component of the body for the motivation of investigation and diagnostic. For finding abnormalities of obtained images normal person information are also maintained in database. MRI is contemplated to the most effective tool for the investigation of the body internal structures among all the above procedure. Among many approaches to detect brain tumor MRI is more efficient. Segmentation is an influential procedure for examine the tumor images effectively. Many segmentation methods are invented [7] such as Watershed, Region Growing Based, Edge Detection Based segmentation and so on.

In clustering and segmentation K-means clustering is popular algorithm which treats each pixel as a feature point having location in space. K-means method randomly choice that cluster number which locates the center of the multidimensional measurement space. The point whose arbitrary mean vector is closest is assigned to the cluster. Continuing this procedure until the remarkable change of the mean vector location between sequential iterations of the algorithms is observed.

The watershed transformation [8] existence is fined by following that a plain surface with few drenches are spilled with water, the water will be filled the deeper gradient before the lighter gradient. In image processing, Watershed algorithm has been used widely in various sectors specially segmentation of medical images. The algorithm is simple, instinctive, faster, adaptable and divides the image completely in disconnected areas though the image shows poor contrast and avoids the requirement for any sort of contour joining [9]. This transformation cannot be affected by lower contrast edges. The local minima are produced because of noise and in the energy minimization process incorrect outcome is acquired.

Compared to the other techniques the segmentation of region based produces segmented regions whose pixel intensity is similar. Seed point election policy is done before the segmentation so that the initial seed point can be selected and the seed point act as a fundamental step for region growing based segmentation [10]. The pixel color, intensity, texture etc. consider as the reference for seed point selection. Once the seed point selection is completed spontaneously region growing taking place.

Morphological operators [11] are known as the nonlinear operators which use to produce a particular shape and structure of the images. This operator works with the pixel order of the image without changing the numerical value. Structuring the element is the main part of this method and it has small matrix of pixel with zero or one value. The matrix dimension is considered for identification of size of structuring element and the matrix dimension is specified by zeros and one pattern. The structuring element is deposit at all potential location of the image and contrast with the corresponding neighbor-hood pixels. Different types of morphological operators such as Dilation, Erosion are employed for image processing. Among all the segmentation process region growing segmentation provide better performance. After segment the brain tumor, the segmented image quality is calculated and MFSIM provides better result. The result of the image quality index identify the distortion level of segmented MRI images and segmented image accuracy level.

\section{Image Quality Assessment}

The quality of image is the fundamental properties of an image through which image degradation is measured by comparing with an ideal or perfect image. The main interesting point here is that the quality of image is reduced by the distortion or artifacts which introduces in imaging systems. The quality of image is defined by the deviation of image from its ideal condition and the measurement of image quality become technical. There have two types of visual quality assessment, one is subjective and the other is objective. Subjective quality assessment is represented the realistic opinion of an image by human being and the objective quality assessment uses the mathematical model. Some common objective image quality assessment is PSNR, MSE and SSIM. Based on the reference image (NR, RR, FR) the image quality assessment is divided in some groups. For image quality measurement testing system, if it has no reference known NR, partial reference known as RR, complete reference known as FR which has complete information so it is shows the best performance of quality prediction. SSIM shows the most existing quality assessment schemes and the great success of SSIM is the adaption of HVS with the structural information. The image visual information often gives redundant value whereas the HVS understand an image which shows low level features like edges and zero-crossings [12]. The reference and distorted image low level feature is compared and obtained IQA metric. Consequently, the FSIM (feature similarity index) which evaluate the image degradation according their feature is 
obtained by FR IQA metric. The image characteristic which is given by the FSIM is improvable. As a result, a novel method is introduced namely Moderate Feature Similarity Index (MFSIM).

\section{A. Feature Similarity Index (FSIM)}

According to the physiological evidence, the point with different frequency of Fourier waves having congruent phases shows visually discernable features. The features with high information are extracted by the high phase congruency (PC) and the PC is the primary feature for the calculation of FSIM. For the computation of the image contrast information image gradient magnitude (GM) which is complementary of PC consider as the secondary feature. Using gray scale images or the luminance components of color images FSIM is designed and for the extension of FSIM chrominance information is used where the extension is called FSIMc.

\section{B. Phase Congruency $(P C)$}

Without direct definition of sharp intensity change features, the PC representation suggest the features point where Fourier components shows maximum value. The dimensionless measurement can be considered by PC and it shows the significant value of local structure.

PC can be showed various implementation techniques for computing the PC mapping of a given image [13]. Consider $\mathrm{g}(\mathrm{x})$ as one dimensional signal, odd and even symmetric filter $\mathrm{M}_{\mathrm{n}}^{\mathrm{e}}$ and $\mathrm{M}_{\mathrm{n}}^{\mathrm{o}}$ respectively on scale $\mathrm{n}$ and form quadratic pair. The response vector at position ax and scale $\mathrm{n}$ is composed by signal quadrature pair response and that response vector is:

$$
\left[e_{n}(x), o_{n}(x)\right]=\left[g(x) * M_{n}^{e}, g(x) * M_{n}^{o}\right]
$$

The local amplitude is:

$$
A_{n}(x)=\sqrt{e_{n}(x)^{2}+o_{n}(x)^{2}}
$$

Let, $\mathrm{F}(\mathrm{x})=\sum \mathrm{e}_{\mathrm{n}}(\mathrm{x})$ and $\mathrm{H}(\mathrm{x})=\sum \mathrm{o}_{\mathrm{n}}(\mathrm{x})$

$$
\mathrm{PC}(\mathrm{x})=\frac{\mathrm{E}(\mathrm{x})}{\epsilon+\sum_{\mathrm{n}} A_{\mathrm{n}}(\mathrm{x})}
$$

Where, $\mathrm{E}(\mathrm{x})=\sqrt{\mathrm{F}(\mathrm{x})^{2}+\mathrm{H}(\mathrm{x})^{2}}$ and $\in$ is a positive constant.

The Gabor and Log-Gabor filter is the main candidate with respect to the filters quadrature i.e. $\mathrm{M}_{\mathrm{n}}^{\mathrm{e}}$ and $\mathrm{M}_{\mathrm{n}}^{\mathrm{o}}[14,15]$. Among these two filters, log-gabor filter is more adaptable and the reason for its more adaptability is given below.

The construction of gabor filter with arbitrary bandwidth is quiet difficult and its maintain low DC component but log-Gabor filter shows absence of DC component.

The log-Gabor filter has high frequency end and extended tail of its transfer function as a result the natural image encoding become more capable [16].

The frequency domain of log-Gabor filter transfer function is:

$$
\mathrm{G}(\omega)=\exp \left(-\frac{\left(\log \left(\omega / \omega_{\mathrm{o}}\right)\right)^{2}}{2 \sigma_{\mathrm{r}}}\right)
$$

Where, $\omega_{\mathrm{o}}=$ center frequency and $\sigma_{\mathrm{r}}=$ controller of bandwidth.

For the computation of PC using 2D gray scale images 1D analysis can be applied in some orientation and the results can be combined by some rule. Using some spreading function, the one-dimension log-Gabor filter can be used as 2D ones. Among different spreading function Gaussian [17] function is one. The combination of 2D log-Gabor and Gaussian spreading function is:

$$
\left.\mathrm{G}_{2}\left(\omega, \theta_{\mathrm{j}}\right)=\exp \frac{\left(\log \left(\omega / \omega_{\mathrm{o}}\right)\right)^{2}}{2 \sigma_{\mathrm{r}}^{2}}\right) * \exp \left(-\frac{\left(\theta-\theta_{\mathrm{j}}\right)^{2}}{2 \sigma_{\theta}^{2}}\right)
$$

Where, the operational angle, $\theta_{\mathrm{j}}=\mathrm{j} \pi / \mathrm{J}, \mathrm{j}=\{0,1, \ldots, \mathrm{J}-1\}, \mathrm{J}=$ number of operations, $\sigma_{\theta}=$ angular bandwidth.

We obtained response after completing modulation between $\omega_{0}$ and $\theta_{j}$ and completing convolution between $G_{2}$ and $2 \mathrm{D}$ image. At each point $\mathrm{x}$ the response is: $\left[\mathrm{e}_{\mathrm{n}, \theta_{\mathrm{j}}}(\mathrm{x}), \mathrm{o}_{\mathrm{n}, \theta_{\mathrm{j}}}(\mathrm{x})\right]$.

For $n$ scale and $\theta_{j}$ orientation the local amplitude is:

$$
A_{n, \theta_{j}}(x)=\sqrt{e_{n, \theta_{j}}(x)^{2}+o_{n, \theta_{j}}(x)^{2}}
$$


And the local energy for $\theta_{\mathrm{j}}$ is:

$$
E_{\theta_{j}}(x)=\sqrt{F_{\theta_{j}}(x)^{2}+H_{\theta_{j}}(x)^{2}}
$$

Where, $F_{\theta_{j}}(x)=\sum_{n} e_{n, \theta_{j}}(x)$ and $H_{\theta_{j}}(x)=\sum_{n} o_{n, \theta_{j}}(x)$.

At point $x$ the $2 \mathrm{D} P C$ is:

$$
\mathrm{PC}_{2 \mathrm{D}}(\mathrm{x})=\frac{\sum_{\mathrm{j}} \mathrm{E}_{\theta_{\mathrm{j}}}(\mathrm{x})}{\epsilon+\sum_{\mathrm{n}} \mathrm{A}_{\mathrm{n}, \theta_{\mathrm{j}}}(\mathrm{x})}
$$

Where $\mathrm{PC}_{2 \mathrm{D}}(\mathrm{x})=$ real number within $0 \sim 1$.

\section{Gradient Magnitude (GM)}

Using convolution masks Gradient operators can be expressed and there are three types of useable gradient operators named Sobel, Prewitt and Scharr operator [18]. Using the above gradient operators in the direction of horizontal and vertical for image the partial derivative is $G_{x}(x)$ and $G_{y}(x)$.

The Sobel operator:

$$
\begin{aligned}
& \mathrm{G}_{\mathrm{x}}(\mathrm{x})=\frac{1}{4}\left[\begin{array}{lllllll}
1 & 0 & -1 ; 2 & 0 & -2 ; 1 & 0 & -1
\end{array}\right]^{*}
\end{aligned}
$$

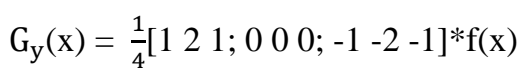

Prewitt operator:

$$
\begin{aligned}
& \mathrm{G}_{\mathrm{x}}(\mathrm{x})=\frac{1}{3}\left[\begin{array}{lllllll}
1 & 0 & -1 ; 1 & 0 & -1 ; 1 & 0 & -1
\end{array}\right]^{*} \mathrm{f}(\mathrm{x}) \\
& \mathrm{G}_{\mathrm{y}}(\mathrm{x})=\frac{1}{3}\left[\begin{array}{lllllll}
1 & 1 & 1 ; 0 & 0 & 0 ;-1 & -1 & -1
\end{array}\right]^{*} \mathrm{f}(\mathrm{x})
\end{aligned}
$$

Scharr operator:

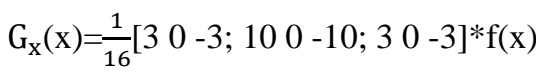

$$
\begin{aligned}
& G_{y}(x)=\frac{1}{16}\left[\begin{array}{lllllll}
3 & 10 & 3 & ; & 0 & 0 & 0
\end{array} ;-3-10-3\right]^{*} f(x)
\end{aligned}
$$

Then the gradient magnitude (GM) is:

$$
G=\sqrt{G_{x}^{2}+G_{y}^{2}}
$$

\section{FSIM Calculation}

FSIM can be calculated using two stages, at first computing the local similarity map then the result of similarity map is pool into the single similarity score. The measurement of feature similarity is divided between two components $f_{1}(x)$ and $f_{2}(x)$, each for PC or GM.

The similarity measurement process using $\mathrm{PC}_{1}(\mathrm{x})$ and $\mathrm{PC}_{2}(\mathrm{x})$ is expressed as:

$$
\mathrm{S}_{\mathrm{PC}}(\mathrm{x})=\frac{\left(2 \mathrm{PC}_{1}(\mathrm{x}) \cdot \mathrm{PC}_{2}(\mathrm{x})+\mathrm{T}_{1}\right)}{\left(\mathrm{PC}_{1}^{2}+\mathrm{PC}_{2}^{2}+\mathrm{T}_{1}\right)}
$$

Where, $\mathrm{T}_{1}=$ positive constant and range $(0,1)$. Similarly, the another expressing for similarity measurement process using $\mathrm{G}_{1}(\mathrm{x})$ and $\mathrm{G}_{2}(\mathrm{x})$ is:

$$
S_{G}(x)=\frac{\left(2 G_{1}(x) \cdot G_{2}(x)+T_{2}\right)}{\left(G_{1}^{2}+G_{2}^{2}+T_{2}\right)}
$$

Where, $\mathrm{T}_{2}=$ positive constant also for GM. 
In all databases $\mathrm{T}_{1}$ and $\mathrm{T}_{2}$ consider to be constant as a result FSIM can be used conveniently. For obtaining the similar nature of $S_{L}(x)$ for $f_{2}(x)$ and $f_{2}(x)$ here combine $S_{P C}(x)$ and $S_{G}(x)$ and definition of $S_{L}(x)$ is:

$$
\mathrm{S}_{\mathrm{L}}(\mathrm{x})=\left[\mathrm{S}_{\mathrm{PC}}(\mathrm{x})\right]^{\alpha} \cdot\left[\mathrm{S}_{\mathrm{PC}}(\mathrm{x})\right]^{\beta}
$$

Where, $\alpha, \beta$ is used for the adjustment of respective importance of PC and GM features.

For the simplicity, $\alpha=\beta=1$.

$$
\text { Thus, } \mathrm{S}_{\mathrm{L}}(\mathrm{x})=\mathrm{S}_{\mathrm{PC}}(\mathrm{x}) * \mathrm{~S}_{\mathrm{G}}(\mathrm{x})
$$

Though the similarity $S_{L}(x)$ can be calculated between $f_{1}$ and $f_{2}$ at each point $x$, HVS has different contribution at different location such as edge location transport. In the structure of phase congruent human visual cortex shows sensitivity [19] as a result the value of PC is reflected like significant structure point. Having a significant PC value for a predefined location $\mathrm{x}$ between $\mathrm{f}_{1}(\mathrm{x})$ and $\mathrm{f}_{2}(\mathrm{x})$ reflects that the position contains high influence of HVS. Therefore, using the function $\mathrm{PC}_{\mathrm{m}}(\mathrm{x})=\max \left(\mathrm{PC}_{1}(\mathrm{x}), \mathrm{PC}_{2}(\mathrm{x})\right)$ the weighted value $\mathrm{S}_{\mathrm{L}}(\mathrm{x})$ is calculated and the FSIM index between $\mathrm{f}_{1}$ and $\mathrm{f}_{2}$ is:

$$
\mathrm{FSIM}=\frac{\sum_{\mathrm{x} \in \Omega} \mathrm{S}_{\mathrm{L}}(\mathrm{x}) \cdot \mathrm{PC}(\mathrm{m})}{\sum_{\mathrm{x} \in \Omega} \mathrm{PC}_{\mathrm{m}}}
$$

Where, $\Omega=$ spatial domain of the whole image.

\section{E. Proposed Methodology}

During evaluating the digital image automatically, sub problems of discovering shapes for example straight line, circles or ellipses are founded. For the detection of edge or the image data some imperfection, missing points or pixels in a specific curve is obtained. The deviation and noise edge points are also showed among ideal line, ellipse or circle. As a result, the extraction of edge features from suitable sets of lines, circles or ellipses is often non-trivial. The Hough transform is addressed the ultimate solution for this problem by making edge groups and that group is obtained executing direct voting system. The Standard Hough Transform (SHT) is obtained from the Hough Function.

The parametric representation of a line using SHT is

$$
r=x^{*} \cos (\theta)+y * \sin (\theta)
$$

Where, $r$ presents perpendicular distance between the origin and the line and $\theta$ represents the angle between the perpendicular projections from the origin to the line and the positive $\mathrm{x}$-axis where the clockwise angle measurement unit is degree. The range of theta is $-90^{\circ} \leq \theta<90^{\circ}$ and the line angle which also measured clockwise form the positive $\mathrm{x}$-axis itself is $\theta+90^{\circ}$.

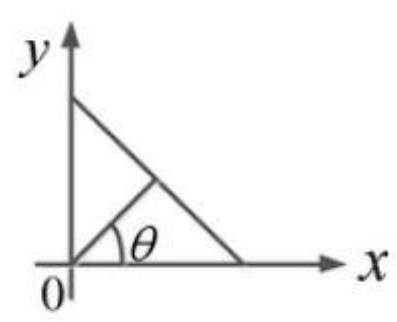

Fig.1. Angle calculation using Hough Transform.

The SHT shows the rho and theta value correspond to rows and column of a space matrix and the elements of SHT constitute accumulator cells. At the beginning each cell shows zero value and the $r$ is calculated for each theta using every single non-background points of image. After the calculation, SHT is rounded $r$ to the nearest row and the increase of accumulator cell is occurred. After completing this operation Q points are founded in the xy-plane by theta and $r$. The potential line of image is presented by peak values of SHT.

In Feature similarity index the parameter theta obtaining form Hough transform can be used. The angular value theta is applied as product with FSIM efficiency increment of the matrices.

For two images $f_{1}(x)$ and $f_{2}(x)$ have separate angle $\theta_{1}(x)$ and $\theta_{2}(x)$. Then the similarity measurement can be defined as,

$$
\mathrm{S}_{\theta}(\mathrm{x})=\frac{\left(2 \theta_{1}(\mathrm{x}) \cdot \theta_{2}(\mathrm{x})+\mathrm{T}_{2}\right)}{\left(\theta_{1}^{2}+\theta_{2}^{2}+\mathrm{T}_{2}\right)}
$$


Where $T_{1}$ and $T_{2}$ are positive constant.

The $\mathrm{S}_{\mathrm{L}}(\mathrm{x})$ can be defined as

$$
\mathrm{S}_{\mathrm{L}}(\mathrm{x})=\left[\mathrm{S}_{\mathrm{PC}}(\mathrm{x})\right]^{\alpha} \cdot\left[\mathrm{S}_{\mathrm{PC}}(\mathrm{x})\right]^{\beta} \cdot\left[\mathrm{S}_{\theta}(\mathrm{x})\right]^{\gamma}
$$

Where, $\alpha, \beta$ is used for adjusting the PC and GM features.

Considering $\alpha=\beta=\gamma=1$.

$$
\text { We get, } S_{L}(x)=S_{P C}(x) \cdot S_{G}(x) \cdot S_{\theta}(x)
$$

Therefore, using the function $\mathrm{PC}_{\mathrm{m}}(\mathrm{x})=\max \left(\mathrm{PC}_{1}(\mathrm{x}), \mathrm{PC}_{2}(\mathrm{x})\right)$ the weighted value $\mathrm{S}_{\mathrm{L}}(\mathrm{x})$ is calculated and the moderated FSIM index introduced as:

$$
\operatorname{MFSIM}=\frac{\sum_{\mathrm{x} \in \Omega} \mathrm{S}_{\mathrm{L}}(\mathrm{x}) \cdot \mathrm{PC} \mathrm{m}(\mathrm{x})}{\sum_{\mathrm{x} \in \Omega} \mathrm{PC}_{\mathrm{m}}}
$$

Where, $\Omega=$ spatial domain of the whole image.

\section{F. Structure Similarity Index}

Structure similarity index is the alternate and complement method image quality assessment problem solution and this process is worked with the basis of top down assumption [20] and good approximation process. The performance of state-of-art for image quality metrics is the simple implementation of structure similarity index [21]. The main disadvantage of SSIM is that it works with single scale only.

Single Scale Structure Similarity Index:

Consider that two non-negative discrete signals aligned with each other one $x=\left\{x_{i} \mid i=1,2\right.$, N\} and the another one is $\left.\mathrm{y}=\mathrm{y}_{\mathrm{i}} \mid \mathrm{i}=1,2, \ldots \ldots \ldots, \mathrm{N}\right\}$ and let the mean and variance of $\mathrm{x}$ is ${ }^{1} \mathrm{x}$ and $3 / 42 \mathrm{x}$ which shows the luminance and contrast respectively and the covariance of $\mathrm{x}$ and $\mathrm{y}$ is $3 / 4 \mathrm{xy}$ which measures the tendency [22]. The equation for the measurement of luminance, contrast and structure comparison is given below:

$$
\begin{array}{r}
1(x, y)=\frac{2 \mu_{x} \mu_{y}+C_{1}}{\mu_{x}^{2}+\mu_{y}^{2}+C_{1}} \\
c(x, y)=\frac{2 \sigma_{x} \sigma_{y}+C_{2}}{\sigma_{x}^{2}+\sigma_{y}^{2}+C_{2}} \\
s(x, y)=\frac{\mu_{x y}+C_{3}}{\sigma_{x} \sigma_{y}+C_{1}}
\end{array}
$$

Where $\mathrm{C}_{1}=\left(\mathrm{K}_{1} \mathrm{~L}\right)^{2}, \quad \mathrm{C}_{2}=\left(\mathrm{K}_{2} \mathrm{~L}\right)^{2}$ and $\mathrm{C}_{3}=\mathrm{C}_{2} / 2$ respectively. $\mathrm{L}=255,8$ bits/pixel and scalar constant $\mathrm{K}_{1} \ll 1$ and $\mathrm{K}_{2} \ll<1$.

Generally, the SSIM is:

$$
\operatorname{SSIM}(x, y)=[1(x, y)]^{\alpha} \cdot[c(x, y)]^{\beta} \cdot[s(x, y)]^{\gamma}
$$

Where $\alpha, \quad \beta$ and $\gamma$ are parameters of three component.

If $\alpha=\beta=\gamma=1$ the resultant SSIM:

$$
\operatorname{SSIM}(x, y)=\frac{\left(2 \mu_{\mathrm{x}} \mu_{\mathrm{y}}+\mathrm{C}_{1}\right)\left(2 \sigma_{\mathrm{x}} \sigma_{\mathrm{y}}+\mathrm{C}_{2}\right)}{\left(\mu_{\mathrm{x}}^{2}+\mu_{\mathrm{y}}^{2}+\mathrm{C}_{1}\right)\left(\sigma_{\mathrm{x}}^{2}+\sigma_{\mathrm{y}}^{2}+\mathrm{C}_{2}\right)}
$$

Multiple Scale Structure Similarity Index:

A single-scale method is applicable for tangible assignment only, on the other hand for different resolution multi-scale approach is more preferable. Consider the original image index scale is 1 , height scale $\mathrm{M}$ and the number of iterations $\mathrm{M}-1$. At the $\mathrm{j}^{\text {th }}$ scale, $c_{j}(x, y)$ and $s_{j}(x, y)$ act as the contrast and structure comparison respectively. $l_{M}(x$, y) act as luminance on $\mathrm{M}$ scale. After completing the combination process among different measurement scale the evaluation of SSIM is achieved and the equation for evaluating SSIM is:

$$
\operatorname{SSIM}(x, y)=\left[l_{M}(x, y)\right]^{\alpha_{M}} \cdot \prod_{j=1}^{M}\left[c_{j}(x, y)\right]^{\beta_{j}} \cdot\left[s_{j}(x, y)\right]^{\gamma_{j}}
$$




\section{G. Universal Image Quality Index (UIQI)}

For the measurement of image and video quality distortion using mathematical model is used UIQI and the model is composed by the combination of correlation, luminance and contrast distortion. Consider two image matric $\mathrm{f}$ and $\mathrm{g}$ with $M$ column and $N$ rows with pixel values $f[i, j], g[i, j]$, respectively $(0 \geq i>M, 0 \geq j>N)$. By completing the combination of three components UIQI is produced.

$$
\mathrm{Q}=\frac{\sigma_{\mathrm{fg}}}{\sigma_{\mathrm{f}} \sigma_{\mathrm{g}}} \frac{2 \overline{\mathrm{fg}}}{(\overline{\mathrm{f}})^{2}+(\overline{\mathrm{g}})^{2}} \frac{2 \sigma_{\mathrm{f}} \sigma_{\mathrm{g}}}{\left(\sigma_{\mathrm{f}}\right)^{2}+\left(\sigma_{\mathrm{g}}\right)^{2}}
$$

Where

$$
\begin{gathered}
\bar{f}=\frac{1}{M N} \sum_{i=0}^{M-1} \sum_{j=0}^{N-1} f[i, j], \quad \bar{g}=\frac{1}{M N} \sum_{i=0}^{M-1} \sum_{j=0}^{N-1} g[i, j] \\
\sigma_{f g}=\frac{1}{M+N-1} \sum_{i=0}^{M-1} \sum_{j=0}^{N-1}(f[i, j]-\bar{f})(g[i, j]-\bar{g}) \\
\left(\sigma_{f}\right)^{2}=\frac{1}{M+N-1} \sum_{i=0}^{M-1} \sum_{j=0}^{N-1}(f[i, j]-\bar{f})^{2} \\
\left(\sigma_{g}\right)^{2}=\frac{1}{M+N-1} \sum_{i=0}^{M-1} \sum_{j=0}^{N-1}(g[i, j]-\bar{g})^{2}
\end{gathered}
$$

The linear correlation rate between $\mathrm{f}$ and $\mathrm{g}$ images is calculated by correlation coefficient of the first component whose variation range is $[-1,1] .1$ is considered as best value and it is obtained by the linear combination of $\mathrm{f}$ and $\mathrm{g}$ that means for all possible values $i$ and $j, g[i, j]=a f[i, j]+b$. The mean luminance is calculated by the $2^{\text {nd }}$ component whose value range is $[0,1]$. The contrast of $\mathrm{f}$ and $\mathrm{g}$ is estimated by the third component and expressed as $\sigma_{\mathrm{f}}$ and $\sigma_{\mathrm{g}}$ and the value range is $[0,1]$. $[-1,1]$ is the range of value for index $\mathrm{Q}$ and the best value is 1 which is obtained for identical images.

\section{H. Peak Signal to Noise Ratio}

For the analysis of images different types of parameters are used. Among them PSNR consider as the important factor for analysis the images. The ratio between the original and noise free images is known peak signal to noise ratio. The equation for calculating PSNR is:

$$
\begin{gathered}
\text { PSNR = } 10 \log _{10}\left(\frac{\mathrm{max}^{2}}{\mathrm{MSE}}\right) \\
=20 \log _{10}\left(\frac{\mathrm{max}}{\sqrt{\mathrm{MSE}}}\right) \\
=20 \log _{10}(\max )-20 \log _{10}(\sqrt{\mathrm{MSE}})
\end{gathered}
$$

Where, MSE $=\frac{1}{\mathrm{mn}} \sum_{\mathrm{t}=0}^{\mathrm{m}-1} \sum_{\mathrm{f}=0}^{\mathrm{n}-1}[\mathrm{l}(\mathrm{t}, \mathrm{f})-\mathrm{k}(\mathrm{t}, \mathrm{f})]^{2}$

\section{Mean Square Error}

The MSE gives value which shows the estimator quality and the value is always non-negative. When the value is more closer to zero it display more accurate value.

The mean-squared error between $l(t, f)$ and $k(t, f)$ is:

$$
\operatorname{MSE}=\frac{1}{\mathrm{mn}} \sum_{\mathrm{t}=0}^{\mathrm{m}-1} \sum_{\mathrm{f}=0}^{\mathrm{n}-1}[\mathrm{l}(\mathrm{t}, \mathrm{f})-\mathrm{k}(\mathrm{t}, \mathrm{f})]^{2}
$$

\section{Image Segmentation}

The image analyzing task can be done subsequently using segmentation process and the performance of the subsequent stages such as classification and feature extraction is affected largely. For the description and recognition of image largely depend on the segmentation outcome. The region is segmented by identifying differences between regions (edges) and common contours. For MRI image segmentation here used Region Growing Segmentation, Watershed Algorithm, K-mean clustering.

\section{A. Threshold the image}

The thresholding is performed with large number of gray level segmentation process using global or local information where binary image is obtained from gray scale image [7]. If the pixel value is below the level of intensity, it is act as white pixel otherwise black. If the image is $\mathrm{f}(\mathrm{x}, \mathrm{y})$, the threshold value is denoted by $\mathrm{T}$. The condition for threshold is: 
if $\mathrm{f}(\mathrm{x}, \mathrm{y})<\mathrm{T}$, then

$\mathrm{f}(\mathrm{x}, \mathrm{y})=255$ or white

otherwise, $\mathrm{f}(\mathrm{x}, \mathrm{y})=0$ or black

In the image processing environment, there exist two types of threshold. One is Global, another is Local.

\section{Global Threshold:}

In threshold process, when the pixels value is above threshold it considers to one otherwise its zero for converting gray-level to binary images. Consider that $\mathrm{g}(\mathrm{x}, \mathrm{y})$ is obtained after completing threshold $\mathrm{f}(\mathrm{x}, \mathrm{y})$ at global threshold $\mathrm{T}$ :

$$
g(x, y)=\left\{\begin{array}{cc}
1 & \text { during } f(x, y) \geq T \\
0 & \text { otherwise }
\end{array}\right.
$$

\section{Local Threshold:}

The illumination change of the image across the image scene causes some part brighter and some part darker in the ways that have nothing to do with the objects images and it is the main problem of the global threshold. In this way the threshold allows smooth variation across the image instead of single global threshold.

\section{B. K-Mean Clustering Algorithm}

The pixels which show similar pattern and characteristics are collected in same group by using this algorithm. The fundamental process of K-Means algorithm [23] is:

a) The center of the cluster is obtained with random manner.

b) The pixels which maintain short distance between center and pixel are applied on the cluster.

c) The cluster pixels are calculated for averaging so that the center of the cluster center can be recomposed.

d) Last two steps are repeated as long as the formation of new cluster center is found.

Working process of K-Means Clustering:

Step 1: after taking the input image it converts into matrix form and save, storing the image size as well.

Step 2: Reshaping the image so that it can present linearly. Step 3: After applying the k-mean into the original input image, at first the cluster or group is formed which have similar feature and the centroids of each group is detected. Step 5: the user input image cluster is numbered and the goal images are clear with the rise of cluster.

Step 6: Most of the centroids of cluster are consider as true value and the remaining centroids consider as false.

Step 7: Then all the dead cells and normal cells are highlighted of the resultant brain image. The normal cells which are not dead are affected because the output images show the defected region.

\section{Problem with K-Means Clustering:}

$\mathrm{K}$-means process is very easy with small complicity but it is unable to show exact result all the time. By the below example this problem can be cleared.

Consider two clusters D and E, two centroids $\mathrm{C} 1$ and $\mathrm{C} 2$ respectively, let object $\mathrm{O}$ present in $\mathrm{D}$ and distance from $\mathrm{C} 1$ is found for $\mathrm{D}$ another distance from $\mathrm{C} 2$ for $\mathrm{E}$ is greater than the distance of $\mathrm{D}$. This algorithm sometimes shows that the object is in the cluster E. But with respect to K-mean algorithm, the object should be in cluster D.

\section{Watershed Algorithm}

The watershed algorithm can be easily described [24]: considering the topographic relief for the image where the gray level of the image is related the height of each point and also considering the gradual fall of rain on the terrain, the lakes (basins) which is formed separated with watershed line. The highest gradient points show the basin boundaries [25] and the original image gradient is computed by watershed algorithm.

\section{Advantages of watershed transform:}

The watershed transform is not affected with edges of low contrast because the significant edges among the markers are found by watershed line. The noise which produces the local minima is found minimum in this technique.

\section{Algorithmic steps for Watershed Segmentation:}

1. The input feature map builds the boundary around the image borders.

2. The input low end feature map shows small fluctuation in the threshold.

3. Each pixel situated into regional minima means the pixels lower valued with respect of its four neighbors located and lave this with integer started with 1. 
4. Each flat section is founded, a label of distance is given, boundary region low pixel is located.

5. The pixel which is not the members of flat region is identified.

6. The unlabeled flat region which is remained is traced with respect to the regional minima. After completing these steps, the label of all the image pixels are completed [26].

7. The flat regions, regional maxima and the boundary pixels are marked.

8. Watershed algorithm computed the regional depth by differentiating the highest and lowest pixel value.

9. The distance finds the threshold value of the image.

10. For creating the segmented image all the marked regions is bounded sequentially.

\section{Problem with Watershed Segmentation:}

The main problem of the watershed algorithm that the local minima show the sensitivity highly and the noisy image influence the segmentation. As a result, the input image is not used directly. Another problem in this method is the over and under segmentation.

\section{Region Growing Segmentation}

Region growing is a segmentation process by which the adjacent pixels are added into a region which has no any edges and the iteration of this process is occurred for every single boundary pixel. This method required a seed point and the growing is started from seed with neighbors which has similar properties. The segmented image is separated from the segmentation process and the remaining pixels select a new seed. The segmentation process largely depends on the initial seed values and the segmentation process lasted till all the pixels are in a segment.

\section{Seed Point Selection:}

In region growing segmentation [27] seed point selection is the initial step which depend on the user and some propertied such as color, texture, pixel intensity etc. For obtaining a seed point the flowing steps need to be performed.

1. The gray scale image is obtained by the conversion of input color image.

2. The pixels whose intensity level is less than or greater than threshold is counted and it is stored using two variables.

3. The difference is calculated using these two variables.

4. Go to step 6 after getting the resultant difference small or conversion is occurred for obtaining negative image, setting zero intensity outside the brain and continuing step 6.

5 . Then the gray image is converted into binary image.

6. The maximum distance between brain part and center is founded.

7. The maximum breadth between rain part and center is obtained and a rectangular shape is obtained by converting the pixels.

8. An array is obtained after storing the sum of all column and row separately.

9. Finally the seed point is taken by the intersection of row and column.

Region Growing Segmentation Algorithm:

The fundamental process for region growing segmentation is [8].

1. $U_{t=1}^{n}=K_{t}=\mathrm{K}$

2. $K_{i}$, connected region, $\mathrm{i}=1,2,3 \ldots, \mathrm{n}$

3. $K_{i} \cap K_{j}=\varphi, \mathrm{i}=1,2,3 \ldots, \mathrm{n}$

4. $\mathrm{P}\left(K_{i}\right)=$ True, $\mathrm{i}=1,2,3 \ldots, \mathrm{n}$

5. $\mathrm{P}\left(K_{i}-K_{j}\right)=$ False, for any adjacent region $K_{i}$ and $K_{j}$

\section{E. Morphological Operator}

When an object need to deformation or reconstruction of shape or structure morphology is used. This operator is applicable for binary images [28] and the use of this operator for filtering, thinning, pruning (pre or post processing) or the shape description or representation. Erosion, Dilation, Opening and Closing is known as the main Morphological operator.

\section{Erosion:}

Consider an image $M$ having two levels zero and one, small structural element (disk, diamond, square) of this image is $\mathrm{Q}$, a pixel $\mathrm{h}$ whose value is changed from one (1) to zero (0). If the predefine value is greater than the central value $\mathrm{h}$ of $\mathrm{Q}$, the resultant value is engaged with the area of $\mathrm{Q}$ i.e. the number of pixels containing value one in the structural element. The requirement of thinning will be obtained by the structural element. 


\section{Dilation:}

In dilation, imagine an image $M$ with two labels one (1) as well as zero (0), $\mathrm{Q}$ is consider as small structural element (disk, diamond, square), $\mathrm{h}$ is a pixel value of $\mathrm{M}$ whose range from one (1) to zero( 0$)$. If the previous determined value is less than the centered value $h$ of $Q$, then the value is assign to zero. The process of growing boundaries in a specific place of image determined the structural element and this is the process of dilation.

$$
\mathrm{M} \oplus \mathrm{Q}
$$

Opening:

The opening operation is performed using two steps one is erosion and another is dilation. Erosion used for removing unwanted pixels and dilation for focusing on the interested area.

$$
\mathrm{M}^{\circ} \mathrm{Q}=(\mathrm{M} \Theta \mathrm{Q}) \oplus \mathrm{Q}
$$

\section{Closing:}

The closing operation also required two steps which is performed in reversing manner of opening. In this process, first step is dilation and second step is erosion using for filing the hole.

$$
\mathrm{M} \bullet \mathrm{Q}=(\mathrm{M} \oplus \mathrm{Q}) \Theta \mathrm{Q}
$$

\section{Result analysis}

There are most commonly useable six databases in IQA community for instance TID2008 [29], CSIQ [22], LIVE, IVC [30], MICT [31] and WIQ [32]. MICT, WIQ and IVC three of them databases which are used for completing this work. For checking validation of the algorithm these three databases are used here. The below table summarize the main feature of these three databases.

Table 1. Bench mark test databases for IQA

\begin{tabular}{|cccccc|}
\hline Database & Source images & Distorted image & Distortion types & Image type & Observer \\
\hline MICT & 14 & 168 & 2 & color & 16 \\
WIQ & 8 & 40 & 4 & gray & unknown \\
IVC & 10 & 185 & 4 & color & 15 \\
\hline
\end{tabular}

One reference and five distorted images are introduced in paper [33] which are denoted below.

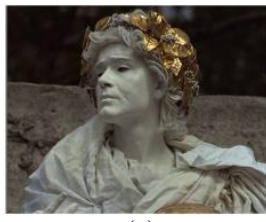

(a)

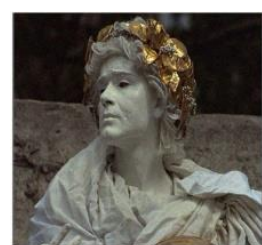

(d)

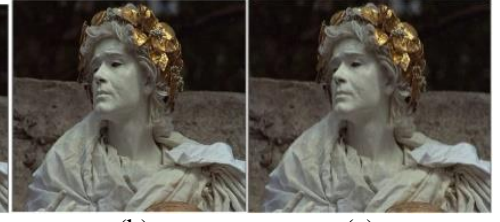

(b)

(c)

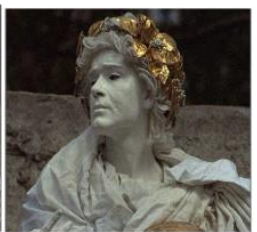

(f)

Fig.2. Reference and distorted images

There used three databases with full reference image. Table 1. shows three databases name IVC, MICT and WIQ. In the table there introduced source images, distorted images, number of observer and image type individually for each database. In Fig.2. image (a) act as reference image and other 5 act as distorted images which is distorted by adding additive Gaussian noise (correlated noise).

The performance of the proposed methodology compare with FSIM is evaluated in tabular format in Table 2. 
Table 2. Compare the performance of proposed method with FSIM.

\begin{tabular}{|l|c|c|c|c|c|l|}
\hline Algorithm & (a) & (b) & (c) & (d) & (f) & Avg. \\
\hline FSIM[29] & 0.9776 & 0.9281 & 0.9827 & 0.9085 & 0.9583 & 0.9509 \\
\hline $\begin{array}{l}\text { Proposed } \\
\text { MFSIM }\end{array}$ & 0.9766 & 0.9633 & 0.9812 & 0.9844 & 0.9844 & 0.9780 \\
\hline
\end{tabular}

From Table 2. we see that the average value of the proposed methodology is .9780 which is greater than the existing system (.9509). If we calculate efficiency, there have $2.7 \%$ more accurate value which is provided by the proposed system.

For evaluating the performance of MFSIM here used four IQA metrics (SSIM, UIQI, PSNR, FSIM). Here also used four performance metrics for the evaluation of IQA metrics. Among them first two (SROCC - Spearman Rank Order Correlation Coefficient, KROCC -Kendal Rank Order Correlation Coefficient) function is used for the measurement of the prediction monotonicity. When the relative distance between two data points ignore and the rank of two data points is needed these two metrics are operated. The regression analysis is applied for computing the other two metrics which is provided by an expert group named VQEG (Video Quality Expert Group) [34]. This regression analysis works with providing the nonlinear mapping between subjective and objective mean opinion scores. After completing nonlinear regression other two metrics (PLCC - Pearson Linear Correlation Coefficient, RMSE - Root Mean Squared Error) is used for linear calculation. The following mapping function can be used for nonlinear regression [35]:

$$
f(x)=\beta_{1}\left(\frac{1}{2}-\frac{1}{1+e^{\beta_{2}\left(x-\beta_{3}\right)}}\right)+\beta_{4} x+\beta_{5}
$$

RMSE value remain small and SROCC, KROCC, PLCC become high which indicate that IQA provide better performance. The performance of IQA metrics summarized in tabular format is given below in Table 3. This table shows that MFSIM provide larger value than all other performance metrics for MICT and WIQ databases with the exception of IVC. So our proposed image quality index provides us better performance for Image quality assessment.

Table 3. Performance analysis of IQA metrics

\begin{tabular}{|c|c|c|c|c|c|c|}
\hline Database & Metrics & MFSIM & SSIM & UIQI & PSNR & FSIM \\
\hline \multirow{5}{*}{ MICT } & SROCC & $\mathbf{0 . 9 1 4 2}$ & 0.8589 & 0.5424 & 0.3445 & 0.9047 \\
& KROCC & $\mathbf{0 . 7 4 6 2}$ & 0.6682 & 0.3845 & 0.2389 & 0.6908 \\
& RMSE & $\mathbf{0 . 8 3 8 3}$ & 0.8086 & 0.7023 & 0.3649 & 0.8275 \\
& RROC & 0.0316 & 0.0313 & .3749 & 0.0438 \\
\hline \multirow{5}{*}{ WIQ } & SROCC & $\mathbf{0 . 5 6 9 8}$ & 0.5250 & 0.5394 & 0.5625 & 0.5549 \\
& KROCC & $\mathbf{0 . 4 4 4 7}$ & 0.3830 & 0.3882 & 0.4062 & 0.4089 \\
& RLCC & $\mathbf{0 . 3 5 0 6}$ & 0.4831 & 0.5842 & 0.6856 & 0.3394 \\
& RMSE & $\mathbf{0 . 5 3 5 6}$ & 0.5381 & 0.5401 & 0.2872 & 0.5439 \\
\hline \multirow{5}{*}{ IVC } & SROCC & 0.3289 & $\mathbf{0 . 6 0 8 4}$ & 0.5088 & 0.0590 & 0.3318 \\
& KROCC & 0.2331 & $\mathbf{0 . 4 3 6 0}$ & 0.3579 & 0.0443 & 0.2048 \\
& PLCC & 0.3616 & $\mathbf{0 . 6 6 9 1}$ & 0.6538 & 0.0491 & 0.3518 \\
& RMSE & 0.0502 & $\mathbf{0 . 7 5 3 3}$ & 0.0502 & 0.4833 & 0.0548 \\
\hline
\end{tabular}



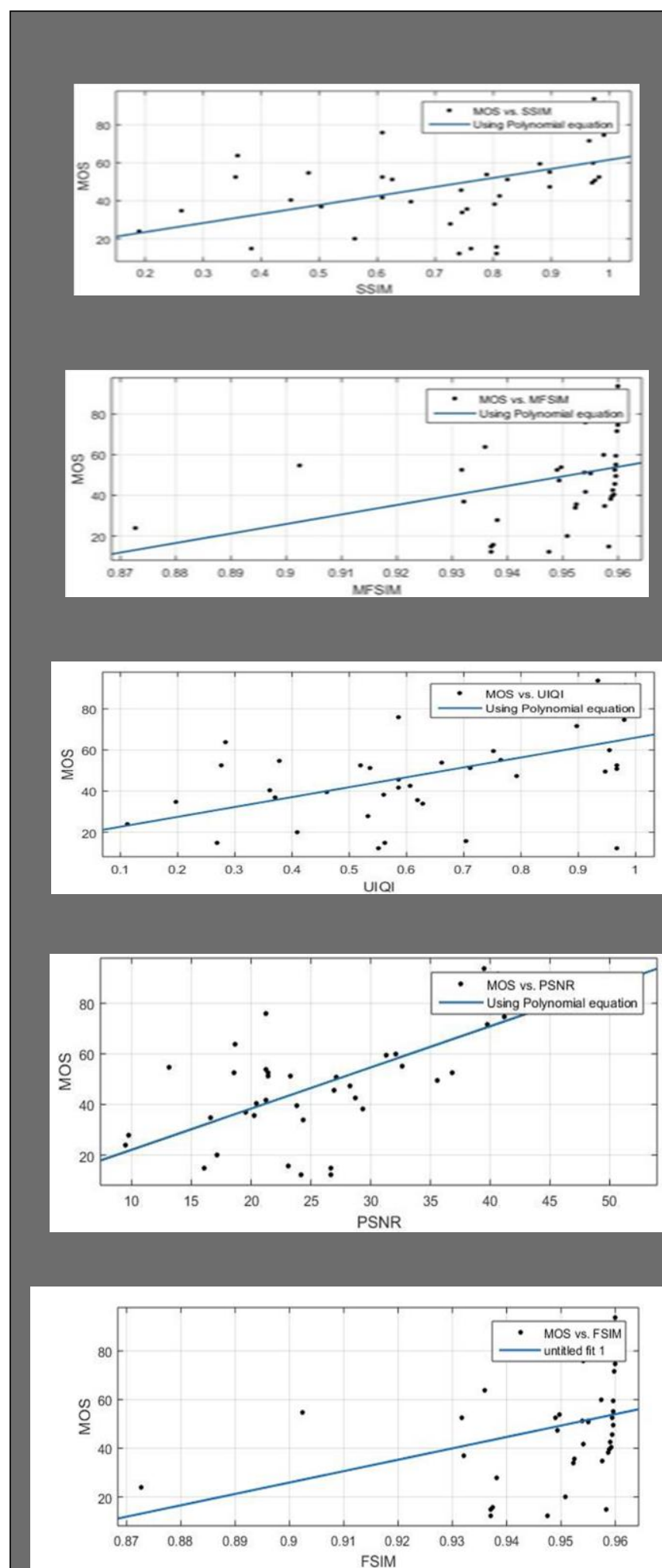

Fig.3. Scattered plot for WIQ database predict the model for achieving subjective MOS versus scores 


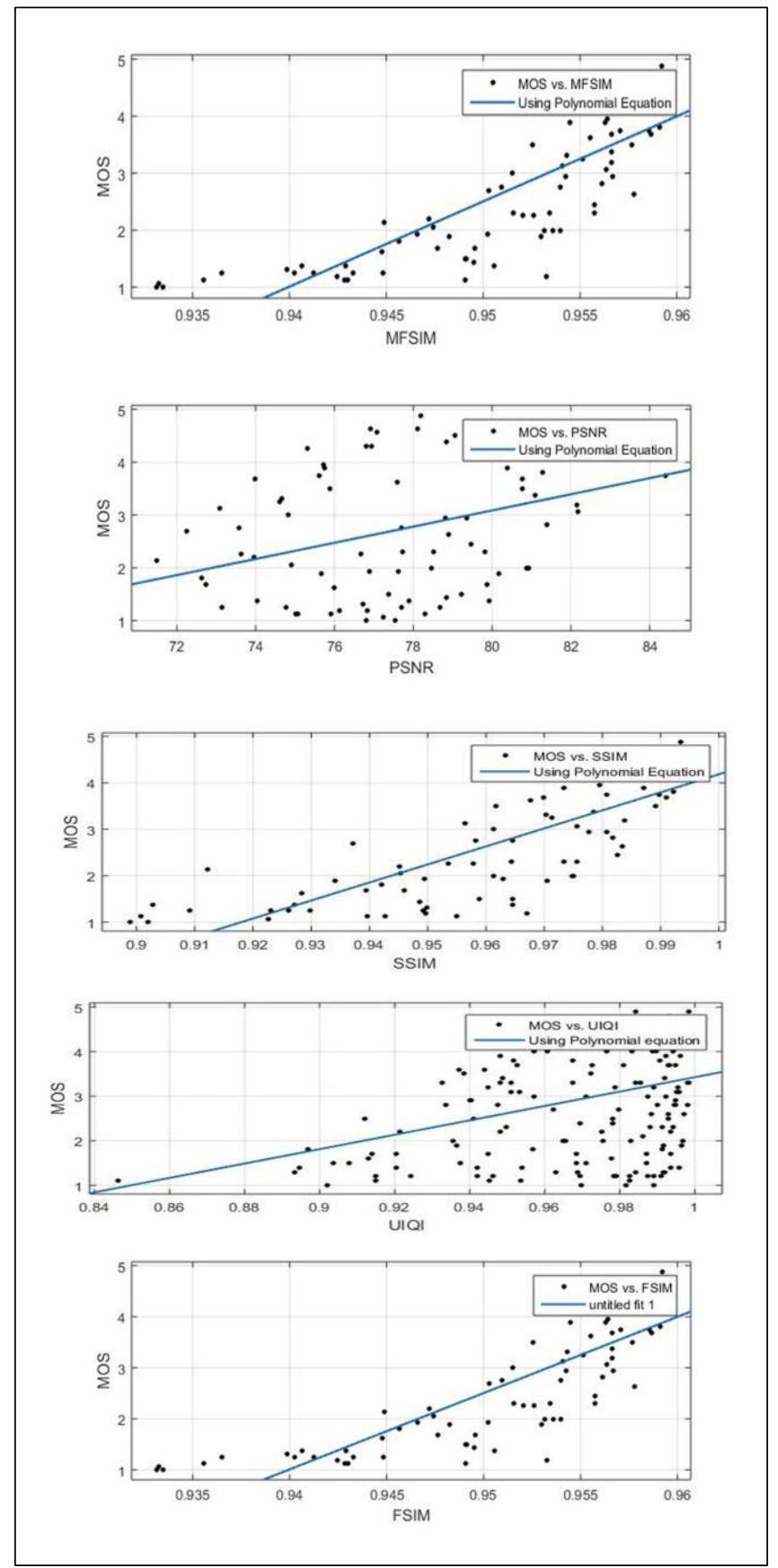

Fig.4. Scattered plot for MICT database predict the model for achieving subjective MOS versus scores.
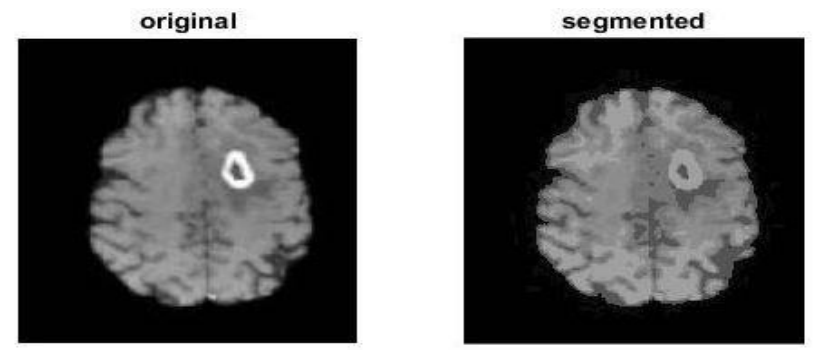

Fig.5. K-mean clustering segmentation output for Tumor 1. 

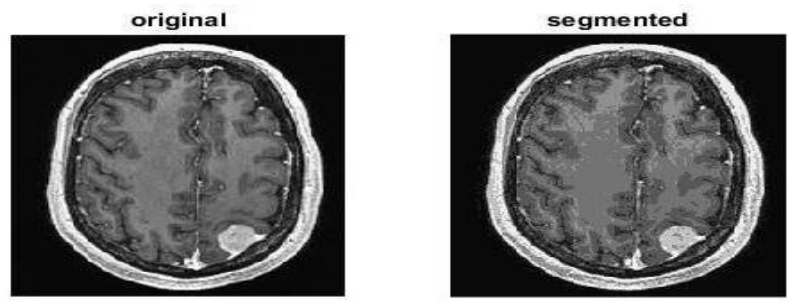

Fig.6. K-mean clustering segmentation output for Tumor 2.
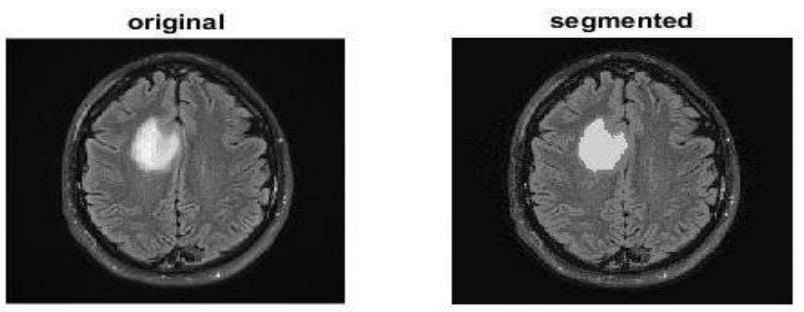

Fig.7. K-mean clustering segmentation output for Tumor 3.
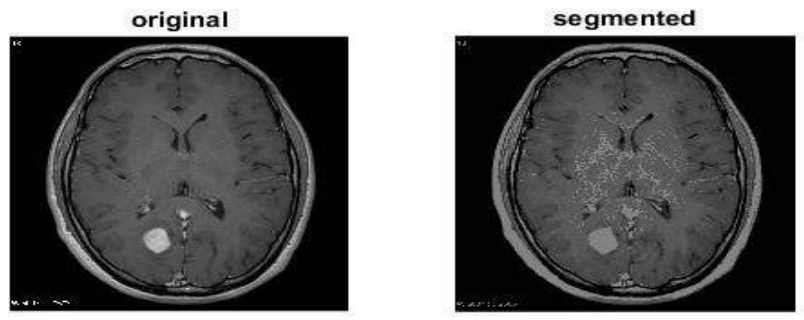

Fig.8. K-mean clustering segmentation output for Tumor 4.

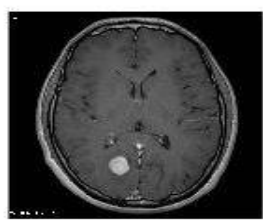

Gradient magnitude (gradmag)
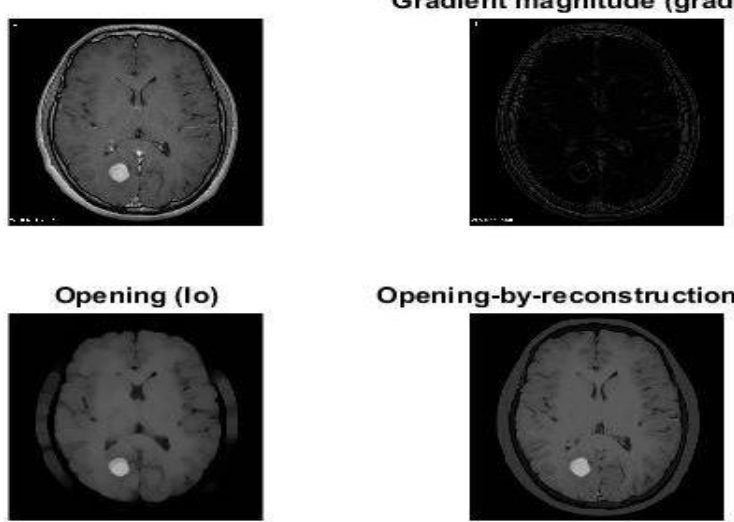

Opening-by-reconstruction (lobr)

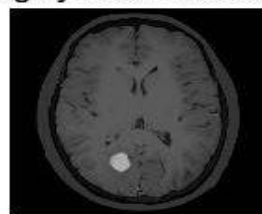

Fig.9. Watershed segmentation output.

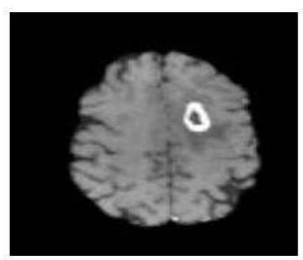

Original image

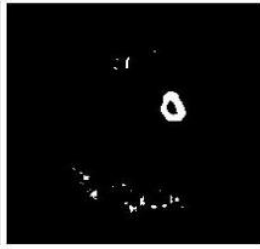

Segmented output

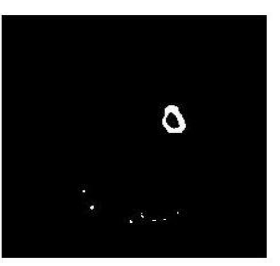

Erosion 


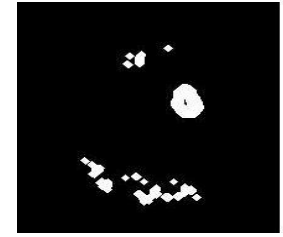

Dilation

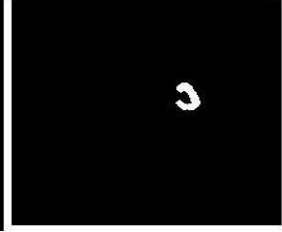

Opening

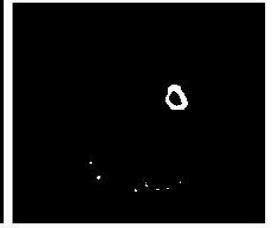

Closing

Fig.10. Region growing segmentation output for Tumor 1.

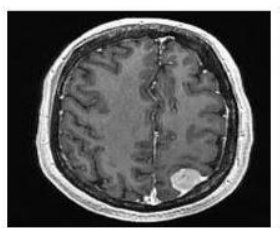

Original image

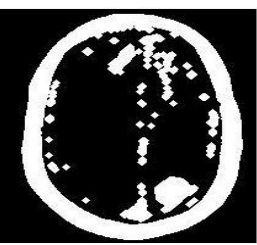

Dilation

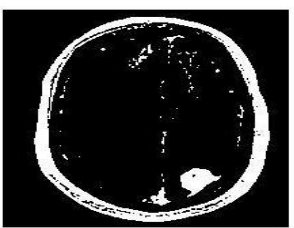

Segmented output

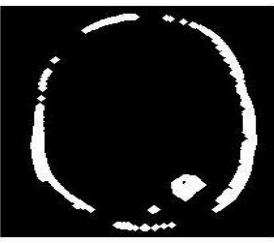

Opening

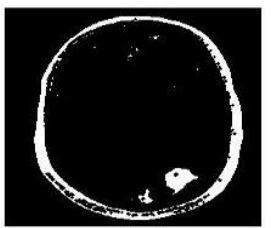

Erosion

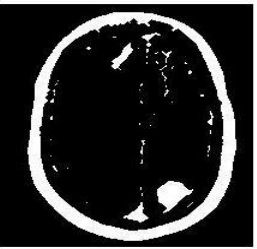

Closing

Fig.11. Region growing segmentation output for Tumor 2.

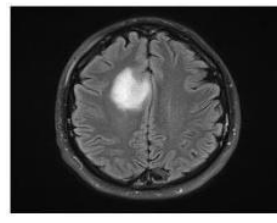

Original image

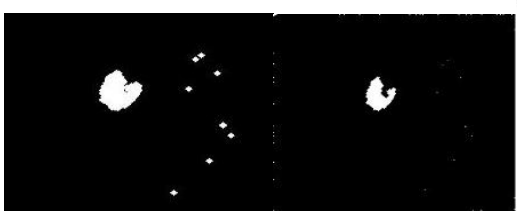

Dilation

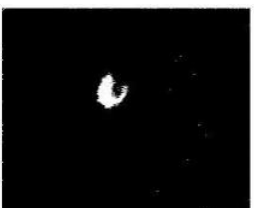

Segmented output

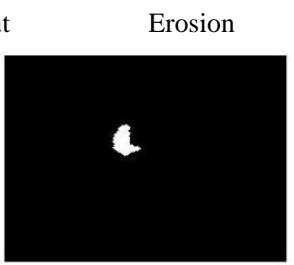

Closing

Fig.12. Region growing segmentation output for Tumor 3.

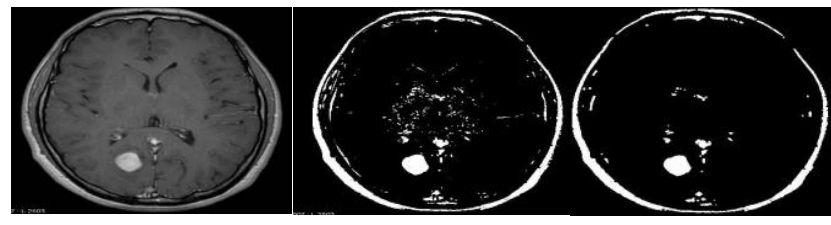

Original image

Segmented output

Erosion

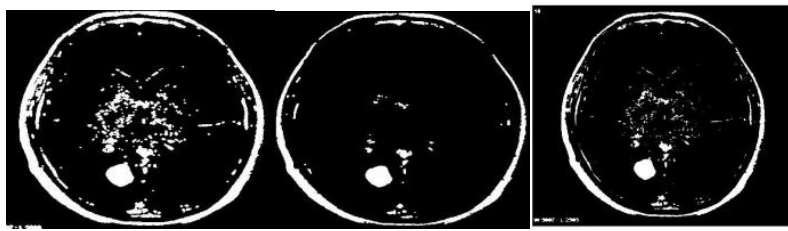

Dilation

Opening

Closing

Fig.13. Region growing segmentation output for Tumor 4. 


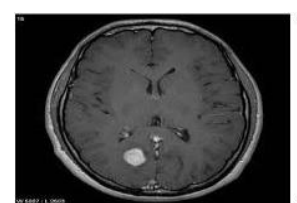

Original image

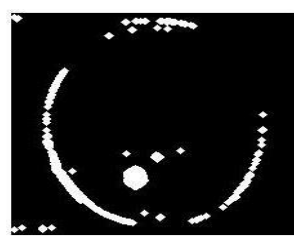

Dilation

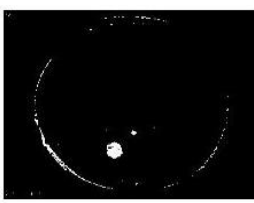

Segmented output

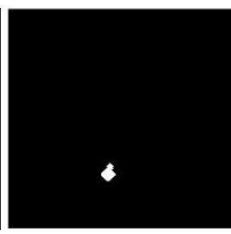

Opening

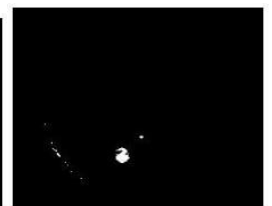

Erosion

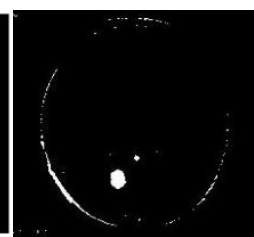

Closing

Fig.14. Region growing segmentation output for Tumor 5.

Table 4. PSNR, MFSIM and FSIM value for Region growing segmentation

\begin{tabular}{|c|c|c|c|}
\hline Tumor Image & PSNR & MFSIM & FSIM \\
\hline Tumor_1 & 37.9990 & $\mathbf{0 . 7 4 6 5}$ & 0.7362 \\
\hline Tumor_2 & 40.0260 & $\mathbf{0 . 3 7 7 2}$ & 0.3250 \\
\hline Tumor_3 & 36.5606 & $\mathbf{0 . 5 2 2 2}$ & 0.5089 \\
\hline Tumor_4 & 36.3015 & $\mathbf{0 . 4 8 6 2}$ & 0.4879 \\
\hline Tumor_5 & 36.2762 & $\mathbf{0 . 5 5 5 5}$ & 0.5461 \\
\hline
\end{tabular}

Table 5. PSNR, MFSIM and FSIM value for Watershed segmentation

\begin{tabular}{|c|c|c|c|}
\hline Tumor Image & PSNR & MFSIM & FSIM \\
\hline Tumor_1 & 19.0945 & $\mathbf{0 . 6 0 6 6}$ & 0.5895 \\
\hline Tumor_2 & 5.0052 & $\mathbf{0 . 2 6 0 0}$ & 0.2752 \\
\hline Tumor_3 & 11.5844 & $\mathbf{0 . 3 7 5 5}$ & 0.3687 \\
\hline Tumor_4 & 17.0650 & $\mathbf{0 . 3 7 9 3}$ & 0.3099 \\
\hline Tumor_5 & 5.5482 & $\mathbf{0 . 3 7 9 3}$ & 0.3802 \\
\hline
\end{tabular}

Table 6. PSNR, MFSIM and FSIM value for K-mean clustering

\begin{tabular}{|c|c|c|c|}
\hline Tumor Image & PSNR & MFSIM & FSIM \\
\hline Tumor_1 & 25.4486 & $\mathbf{0 . 9 5 4 7}$ & 0.9057 \\
\hline Tumor_2 & 24.7719 & $\mathbf{0 . 9 5 3 7}$ & 0.8992 \\
\hline Tumor_3 & 30.2530 & $\mathbf{0 . 9 5 5 6}$ & 0.9504 \\
\hline Tumor_4 & 22.3145 & $\mathbf{0 . 9 5 4 2}$ & 0.9438 \\
\hline Tumor_5 & 25.7311 & $\mathbf{0 . 9 5 4 9}$ & 0.9359 \\
\hline
\end{tabular}

Table 4., Table 5. and Table 6. Show the result for individual segmentation Region growing, Watershed and K-mean clustering segmentation process respectively. For all the method here used five same tumor so that we can easily compare the result. We used three image quality index PSNR, FSIM and MFSIM and we see from all three table MFSIM which is our proposed image quality measurement index provides more accurate result than FSIM. 
Table 7. Performance comparison for image segmentation methods.

\begin{tabular}{|c|c|c|c|}
\hline $\begin{array}{c}\text { Segmentation } \\
\text { algorithm }\end{array}$ & PSNR & MFSIM & FSIM \\
\hline Region Growing & 37.4266 & $\mathbf{0 . 5 3 7 5}$ & 0.5208 \\
\hline Watershed & 11.6595 & $\mathbf{0 . 4 0 0 1}$ & 0.3847 \\
\hline K-mean & 25.7038 & $\mathbf{0 . 9 5 4 6}$ & 0.9273 \\
\hline
\end{tabular}

Table 7. represents the average performance value for all the segmentation method. The PSNR, MFSIM and FSIM values for Region growing based segmentation is $37.4266,0.5375$ and 0.5208 respectably, for Watershed segmentation algorithm is $11.6595,0.4001$ and 0.3847 respectably, for K-mean clustering segmentation algorithm is $25.7038,0.9546$ and 0.9273 respectably. Form the Table 7 . We see that the PSNR value for region growing segmentation is higher than the other two segmentations which indicate that region growing segmentation is better than the other segmentation. In order to measure the image quality assessment, the segmented image is tested using different image quality index. We see that the average IQA value of our proposed method MFSIM is 0.631 whereas FSIM is 0.611 . Our proposed method provides us $3.27 \%$. better performance than the existing method.

\section{Conclusion}

The main target of this research work is to find out an authentic image quality index which provides us better performance than the existing method. We introduced a techniques named Moderate FSIM which used the third component of image namely phase with first two component magnitude and frequency whereas the existing system used only first two component of image. In order to develop our system we used three databases (MICT, WIQ, IVC). We used additional existing image quality index like SSIM, PSNR, UIQI, FSIM so that we can compare our proposed index result perfectly. From Table 2. and Table 3. in the result analysis section, we see that our proposed index provide better performance than the other index and it provides exactly $2.71 \%$ better performance than FSIM. Another objectives of this work is the detect brain tumor for MRI image using segmentation method and analysis the quality of segmented image using our proposed image quality index. For brain tumor detection we used three segmentation techniques and we see that all the techniques segment the image perfectly. In order to testing the quality of the segmented image, we applied these images to our proposed index and existing index. From Table 7. we see that the PSNR value of Region growing segmentation is higher than the other two segmentation, so we can comment that region growing segmentation provides better performance than the other two segmentation techniques. We also see that from same table (Table 7.) our proposed image quality index (MFSIM) display lager value for all the segmentation method than the existing image quality index (FSIM). The proposed index provides exactly $3.27 \%$ more accurate result. From the above analysis it is clear that the brain tumor is segmented successfully and calculates the quality of image properly. We also clear that our proposed image quality index gives us better performance than the other index. This research work mainly advanced the measurement process of image quality then existing method. In future we try to use more database like TID, CSIQ and LIVE. We try to find out the shape and area of the tumor in future.

\section{References}

[1] Z. Wang, A.C. Bovik, H.R. Sheikh, and E.P. Simoncelli, "Image quality assessment: from error visibility to structural similarity”, IEEE Trans. Image Process., vol. 13, no. 4, pp. 600-612, Apr. 2004.

[2] N. Damera-Venkata, T.D. Kite, W.S. Geisler, B.L. Evans and A.C. Bovik, "Image quality assessment based on a degradation model”, IEEE Trans. Image Process., vol. 9, no. 4, pp. 636-650, Apr. 2000.

[3] H.R. Sheikh and A.C. Bovik, "Image information and visual quality", IEEE Trans. Image Process., vol. 15, no. 2, pp. 430-444, Feb. 2006.

[4] Sheikh Md. Rabiul Islam; Md. Tariqul Islam; Xu Huang, “A new approach of image quality index” Advances in Electrical Engineering (ICAEE), 2017 4th International Conference on, Dhaka, Bangladesh, pp.223-228, 28-30 September, 2017.

[5] Z. Wang, E.P. Simoncelli, and A.C. Bovik, "Multi-scale structural similarity for image quality assessment", presented at the IEEE Asilomar Conf. Signals, Systems and Computers, Nov. 2003.

[6] D. Marr, Vision. New York: W. H. Freeman and Company, 1980.

[7] Natarajan, Krishnan, Natasha Saneep Kenkre," Tumor Detection using threshold operation in MRI Brain Images" International Conference on Computer intelligence and Computing Reseach, pp 1-4, 2012.

[8] Nassir Salman," Image Segmentation Based on Watershed and Edge Detection Techniques", The International Arab Journal of Information Technology, vol 3,pp 104-110,2006.

[9] S. Armato III, M. Giger, and H. MacMahon, "Automated detection of lung nodules in CT scans: Preliminary result," Med. Phys. 28, 1552-1561_2001_. 
[10] Yu-Li You and M. Kaveh, "Fourth order partial differential equations for noise removal," IEEE Trans. Image Processing, Vol. 9, No. 10, 2000, pp 1723-1730.

[11] H. S. Prashanta, Dr. Shahidhara. H.L. Dr. K.N.B. Murthy Madhavi Lata. G. "Medical Image Segmentation" (IJCSE) International Journal on Computer Science and Engineering, Vol. 02, 2010, No.04, 1209-1218.

[12] M.C. Morrone and D.C. Burr, "Feature detection in human vision: a phase-dependent energy model", Proc. R. Soc. Lond. B, vol. 235, no. 1280, pp. 221-245, Dec. 1988.

[13] M.C. Morrone, J. Ross, D.C. Burr, and R. Owens, "Mach bands are phase dependent", Nature, vol. 324, no. 6049, pp. 250-253, Nov. 1986.

[14] D. Gabor, "Theory of communication”, J. Inst. Elec. Eng., vol. 93, no. III, pp. 429-457, 1946.

[15] D. J. Field, "Relations between the statistics of natural images and the response properties of cortical cells", J. Opt. Soc. Am. A, vol. 4, no. 12, pp. 2379-2394, Dec. 1987.21

[16] P. Kovesi, "Image features from phase congruency", Videre: J. Comp. Vis. Res., vol. 1, no. 3, pp. 1-26, 1999.

[17] W. Wang, J. Li, F. Huang, and H. Feng, "Design and implementation of log-Gabor filter in fingerprint image enhancement", Pattern Recognit. Letters, vol. 29, no. 3, pp. 301-308, Feb. 2008.

[18] B. Jähne, H. Haubecker, and P. Geibler, Handbook of Computer Vision and Applications. Academic Press, 1999.

[19] L. Henriksson, A. Hyvärinen, and S. Vanni, "Representation of cross-frequency spatial phase relationships in human visual cortex", J. Neuroscience, vol. 29, no. 45, pp. 14342-14351, Nov. 2009.

[20] Z. Wang and A. C. Bovik, “A universal image quality index,” IEEE Signal Processing Letters, vol. 9, pp. 81-84, Mar. 2002.

[21] Z. Wang, H. R. Sheikh, and A. C. Bovik, "Objective video quality assessment," in The Handbook of Video Databases: Design and Applications (B. Furht and O. Marques, eds.), pp. 1041-1078, CRC Press, Sept. 2003

[22] E.C. Larson and D.M. Chandler, "Categorical Image Quality (CSIQ) Database".

[23] M. Masroor Ahmed. Dzulkifli Bin Mohammad, " Segmentation of Brain MR Images for Tumor Extraction by Combining Kmeans Clustering and Perona-Malik Anisotropic Diffusion Model", IEEE International Journal of Image Processing,pp27-34,2011

[24] S. Beucher and F. Meyer, "The morphological approach to segmentation: The watershed transform," in Mathematical Morphology in Image Processing, E. R. Dougherty, Ed. New York: Marcel Dekker, 1993, vol. 12, pp. 433-481.

[25] S. Armato III, M. Giger, and H. MacMahon, "Automated detection of lung nodules in CT scans: Preliminary result," Med. Phys. 28, 1552-1561_2001_.

[26] R. Haralick, "Statistical and structural approaches to texture," Proc. IEEE 67, 786-804_1979_.

[27] Swati Tiwari, Ashish Bansal and Rupali Sagar, "Identification of brain tumors in 2D MRI using Automatic seeded region growing”, Journal of Education, vol 1, issue 2,2012.

[28] Nursuriati Jamil, Tengku Mohd Tengku, Zainab Bakar," Noise Removal and Enhancement of Binary Images Using Morphological Operations" IEEE International Symposium on Information Technology, pp1-6,2008

[29] N. Ponomarenko, V. Lukin, A. Zelensky, K. Egiazarian, M. Carli, and F. Battisti, “ TID2008 - A database for evaluation of full-reference visual quality assessment metrics", Advances of Modern Radioelectronics, vol. 10, pp. 30-45, 2009.

[30] Ninassi, P. Le Callet, and F. Autrusseau, "Subjective quality assessment-IVC database".

[31] Y. Horita, K. Shibata, Y. Kawayoke, and Z.M. Parves Sazzad, "MICT Image Quality Evaluation Database".

[32] D.M. Chandler and S.S.Hemami, "A57 database".

[33] A Feature Similarity Index for Image Quality Assessment Lin Zhang, Student Member, IEEE, Lei Zhang, Member, IEEE, Xuanqin Mou, Member, IEEE, and David Zhang, Fellow, IEEE

[34] C. Yang and S.H. Kwok, "Efficient gamut clipping for color image processing using LHS and YIQ", Optical Engineering, vol. 42, no. 3, pp.701-711, Mar. 2003.

[35] M.C. Morrone and R.A. Owens, "Feature detection from local energy", Pattern Recognit. Letters, vol. 6, no. 5, pp. 303-313, Dec. 1987.

\section{Authors' Profiles}

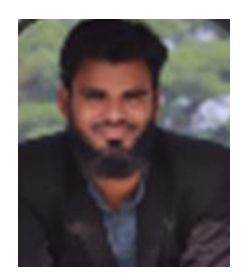

Md. Tariqul Islam received the B.Sc. Engineering (ECE) degree form Khulna University of Engineering and Technology (KUET), Bangladesh, June 2017. He is currently working as a Lecturer in Department of Computer Science and Engineering (CSE) in Bangladesh University, Dhaka, Bangladesh where he joined in January, 2018. His main research interest includes Biomedical Signal and Image processing, Image processing, Machine learning and Deep Learning. He has published three international conference papers.

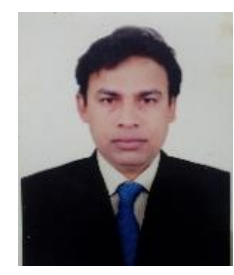

Sheikh Md. Rabiul Islam received the B.Sc. in Engineering (ECE) from Khulna University, Khulna, Bangladesh in December 2003, and M.Sc. in Telecommunication Engineering from the University of Trento, Italy, in October 2009 and Ph.D. from University of Canberra, Australia, in 2015. He joined as a Lecturer in the department of Electronics and Communication Engineering of Khulna University of Engineering \& Technology, Khulna, in 2004, where he is joined an Assistant Professor in the same department in the effect of 2008. Also he joined Associate Professor in 2015 and joined as Professor in 2018 in the same department. He has given four M.Sc. Engineering at Dept. of ECE and one M.Sc. Engineering (BME) at Dept of Biomedical Engineering. He is 
also completed four projects by funded university grant commission. Now He has published 2 book chapters, 30 Journal and 33 International conferences. His research interests include biomedical signal \& image processing, VLSI for Biomedical signal and image processing, Machine learning, Embedded system. He is an IEEE member.

How to cite this paper: Md. Tariqul Islam, Sheikh Md. Rabiul Islam, " A New Image Quality Index and it's Application on MRI Image", International Journal of Image, Graphics and Signal Processing(IJIGSP), Vol.13, No.4, pp. 14-32, 2021.DOI: 10.5815/ijigsp.2021.04.02 